\title{
Mapping Resonance Structures in Transient Core-Ionized Atoms
}

T. Mazza $\odot,{ }^{1}$ M. Ilchen, ${ }^{1,2}$ M. D. Kiselev $\odot,{ }^{3,4}$ E. V. Gryzlova $\odot,{ }^{3}$ T. M. Baumann, ${ }^{1}$ R. Boll, ${ }^{1}$ A. De Fanis, ${ }^{1}$ P. Grychtol, ${ }^{1}$ J. Montaño, ${ }^{1}$ V. Music, ${ }^{1,2}$ Y. Ovcharenko, ${ }^{1}$ N. Rennhack, ${ }^{1}$ D. E. Rivas, ${ }^{1}$ Ph. Schmidt, ${ }^{1,2}$ R. Wagner, ${ }^{1}$ P. Ziolkowski, ${ }^{1}$, N. Berrah, ${ }^{5}$ B. Erk, ${ }^{6}$ P. Johnsson, ${ }^{7}$ C. Küstner-Wetekam, ${ }^{2}$ L. Marder, ${ }^{2}$ M. Martins, ${ }^{8}$ C. Ott $\odot,{ }^{9}$ S. Pathak, ${ }^{10}$ T. Pfeifer, ${ }^{9}$ D. Rolles, ${ }^{10}$ O. Zatsarinny, ${ }^{11}$ A. N. Grum-Grzhimailo $\odot{ }^{3}$ and M. Meyer ${ }^{1}$

${ }^{1}$ European XFEL, Holzkoppel 4, 22869 Schenefeld, Germany

${ }^{2}$ Institute of Physics, University of Kassel, Heinrich-Plett-Strasse 40, 34132 Kassel, Germany

${ }^{3}$ Skobeltsyn Institute of Nuclear Physics, Lomonosov Moscow State University, 119991 Moscow, Russia

${ }^{4}$ Faculty of Physics, Lomonosov Moscow State University, 119991 Moscow, Russia

${ }^{5}$ Physics Department, University of Connecticut, Unit 3046, Storrs, Connecticut 06269-3046, USA

${ }^{6}$ Deutsches Elektronen-Synchrotron DESY, Notkestrasse 85, 22607 Hamburg, Germany

${ }^{7}$ Department of Physics, Lund University, Box 117, 22100 Lund, Sweden

${ }^{8}$ Institute of Experimental Physics, University of Hamburg, Luruper Chaussee 149, 22761 Hamburg, Germany

${ }^{9}$ Max-Planck-Institut für Kernphysik, Saupfercheckweg 1, 69117 Heidelberg, Germany

${ }^{10}$ J.R. Macdonald Laboratory, Department of Physics, Kansas State University, 116 Cardwell Hall, Manhattan, Kansas 66506-2601, USA

${ }^{11}$ Department of Physics and Astronomy, Drake University, Des Moines, Iowa 50311, USA

(Received 8 June 2020; revised 5 September 2020; accepted 8 October 2020; published 18 December 2020)

The nature of transient electronic states created by photoabsorption critically determines the dynamics of the subsequently evolving system. Here, we investigate $K$-shell photoionized atomic neon by absorbing a second photon within the Auger-decay lifetime of 2.4 fs using the European XFEL, a unique highrepetition-rate, wavelength-tunable x-ray free-electron laser. By high-resolution electron spectroscopy, we map out the transient Rydberg resonances unraveling the details of the subsequent decay of the hollow atom. So far, ultra-short-lived electronic transients, which are often inaccessible by experiments, were mainly inferred from theory but are now addressed by nonlinear x-ray absorption. The successful characterization of these resonances with femtosecond lifetimes provides the basis for a novel class of sitespecific, nonlinear, and time-resolved studies with strong impact for a wide range of topics in physics and chemistry.

DOI: $10.1103 /$ PhysRevX.10.041056

Subject Areas: Atomic and Molecular Physics

\section{INTRODUCTION}

Transient electronic states play a crucial role in complex physical or chemical processes in atoms [1,2] and molecules [3-7], as well as photocatalysis [8,9], elementary steps in photosynthesis [10], and radiation damage [11]. Their lifetimes are governed by various processes that take place on a broad range of timescales. For the fastest of these processes, many photon-mediated electronic processes are often the first step to relaxation dynamics, energy transfer, molecular restructuring, or dissociation, and can constitute the beginning of a structure-function interplay that is in the center of ultrafast time-resolved investigations. However,

Published by the American Physical Society under the terms of the Creative Commons Attribution 4.0 International license. Further distribution of this work must maintain attribution to the author(s) and the published article's title, journal citation, and DOI. due to their short lifetimes, transient states often escape experimental observation. X-ray free-electron lasers (XFELs) being the most brilliant $\mathrm{x}$-ray sources in the world can provide up to $10^{14}$ photons per pulse, i.e., within exposure times at the timescale of atomic and even electronic dynamics, in femto- and attoseconds, respectively. In addition to providing the required time resolution for recording snapshot sequences of ultrafast processes at the atomic scale, they are suitable tools for creating, exploiting, and investigating transient structures and resonances, while giving access to site-specific information by element-specific core excitations in the soft x-ray regime [12]. At these short wavelengths, the first step of lightmatter interaction is, predominantly, the photoemission of a core electron that alters the Coulombic potential of the targeted system within attoseconds. Such a core-ionized system typically decays within a few femtoseconds, which subsequently triggers a variety of processes. Probing the electronic structure of a core-excited system before any relaxation occurs therefore provides essential information 
for a better understanding of ultrafast processes, nonlinear photoabsorption at extreme intensities, and in particular, radiation damage in FEL-based imaging experiments. Moreover, transient resonances play a substantial role in multiple-ionization processes at FELs, not only at the beginning, but also during each step of the interaction with the ultraintense $\mathrm{x}$-ray pulse [1-3]. Experimentally addressing these elusive structures can help to validate the latest theoretical understanding of complex transient states in their role as mediators of photoinduced excitation processes, which is promising to support advanced applications in physics, chemistry, and biology [1-12].

\section{RESULTS AND DISCUSSION}

\section{A. The process}

In this work, we use high-resolution electron spectroscopy available at the Small Quantum Systems (SQS) instrument of the European XFEL to experimentally characterize transient core-ionized neon atoms within their lifetimes of only approximately 2.4 fs $[13,14]$. This method has been shown to be a highly differential tool for investigating electronic structures and dynamics of ionic matter [15-17]. By applying resonant Auger spectroscopy, we succeed in mapping the high-lying $n p$-Rydberg resonances of the transient $\mathrm{Ne}^{+} 1 s^{-1}$ ion by populating them with the remaining electron from the core level by excitations of the type $\mathrm{Ne}^{+} 1 s^{1} 2 s^{2} 2 p^{6}+h \nu \rightarrow$ $\mathrm{Ne}^{+*} 1 s^{0} 2 s^{2} 2 p^{6} n p$ (Fig. 1).

Neon has a $1 s$ electron binding energy of $870.2 \mathrm{eV}$, which is giving the minimal energy requirement for one photon to generate a single-core hole ( $\mathrm{SCH})$. After ionization, the remaining core electron becomes more tightly bound by about $125 \mathrm{eV}$. Without further interaction, the stored Coulombic energy in the system would be released within approximately $2.4 \mathrm{fs}$ via Auger decay. If another photon with sufficient energy is absorbed before the ultrafast relaxation, the remaining electron from the core orbital can be ejected or resonantly excited into a valence orbital, thus creating a (resonant) double-core hole (DCH) [18] (see Fig. 1).

By mapping out the resonances in such a highly excited and transient system, the ion structure after core-hole generation can be studied before relaxation takes place. Therefore, the presented method can create an observation window into the very beginning of subsequently evolving $\mathrm{x}$-ray-induced dynamics. This is complementary to the concept of the core-hole clock $[19,20]$, which uses the decay of the short-lived state to obtain information on the dynamics within the lifetime of the core-hole state. The formation of $\mathrm{DCH}$ provides access to the time window even before this decay.

\section{B. Experimental results}

The present study exploits the possibilities given by the smooth wavelength tunability and the high repetition rate for the $\mathrm{x}$-ray pulse delivery at the European XFEL, the first

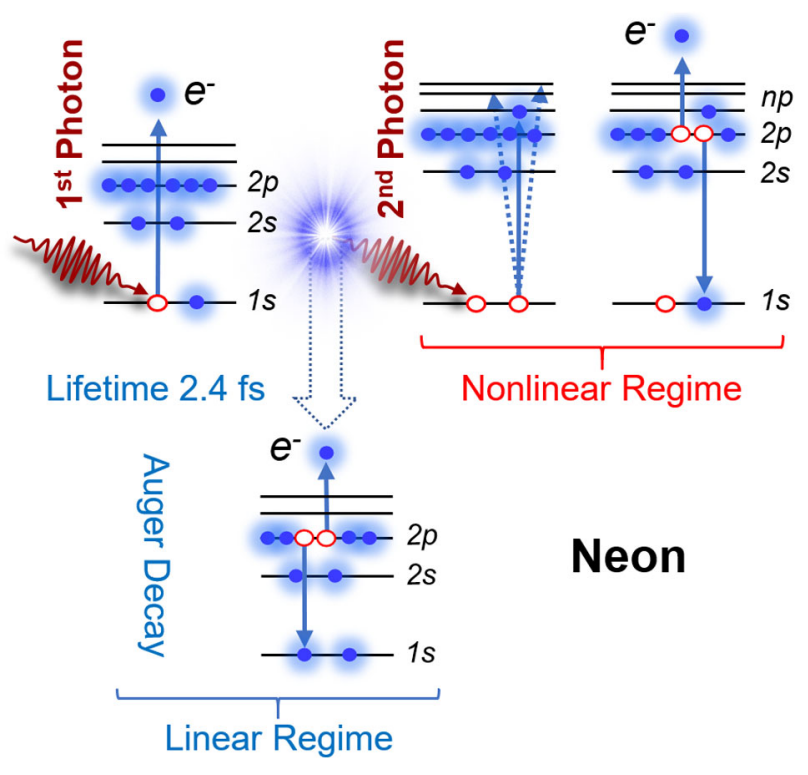

FIG. 1. Scheme of the investigated process. Linear regime (left): The first photon absorption and resulting ionization of neon is typically followed by an Auger decay after approximately 2.4 fs (from top to bottom). Nonlinear regime (right): With short pulses of sufficiently high fluence, this sequence can be intercepted by a second photon that creates $1 s^{-2} n p$-Rydberg states in the transient ion. The relaxation of this double-core hole results in the ejection of an electron, which is the subject of the present investigation.

XFEL entering an effective $\mathrm{kHz}$ regime [21] (up to $2 \mathrm{kHz}$ used in the present experiment). The ultrashort, intense $\mathrm{x}$-ray pulses of about $2 \mathrm{~mJ}$ energy and pulse durations of about 25 fs are focused to about $1.5 \times 1.5 \mu \mathrm{m}^{2}$ (FWHM) via Kirkpatrick-Baez (KB) optics on neon atoms in the acceptance volume of a time-of-flight electron spectrometer within an ultra-high-vacuum chamber (see Sec. IV.).

The success of the experimental observation of these transient resonances depends in a crucial way on the ratio between the pulse duration and the $\mathrm{SCH}$ lifetime; in addition, it depends on the ratio between the bandwidth of the exciting x-ray pulse and the spectral width of the DCH resonances. For our experimental conditions, these ratios are independent from each other, as the spectral and temporal properties of self-amplified spontaneous emission (SASE) pulses are far from the Fourier limit. They are given by $25 \mathrm{fs}$ (pulse duration) vs $2.4 \mathrm{fs}$ (lifetime) and $8.5 \mathrm{eV}$ (XFEL SASE bandwidth) vs $0.7 \mathrm{eV}$ (resonance width), respectively, affecting the ratio of the $\mathrm{DCH}$ and $\mathrm{SCH}$ yields, which in the perturbative regime depends linearly on the intensity (see Sec. IV). Irrespective of the number of photons irradiating the sample, it results already in a more than 2 orders of magnitude lower probability of absorbing a second photon compared to the first one, assuming that the cross sections for the absorption of the two photons are comparable. In fact, at any intensity the absorption of a second photon, when present, can occur only if the photon 


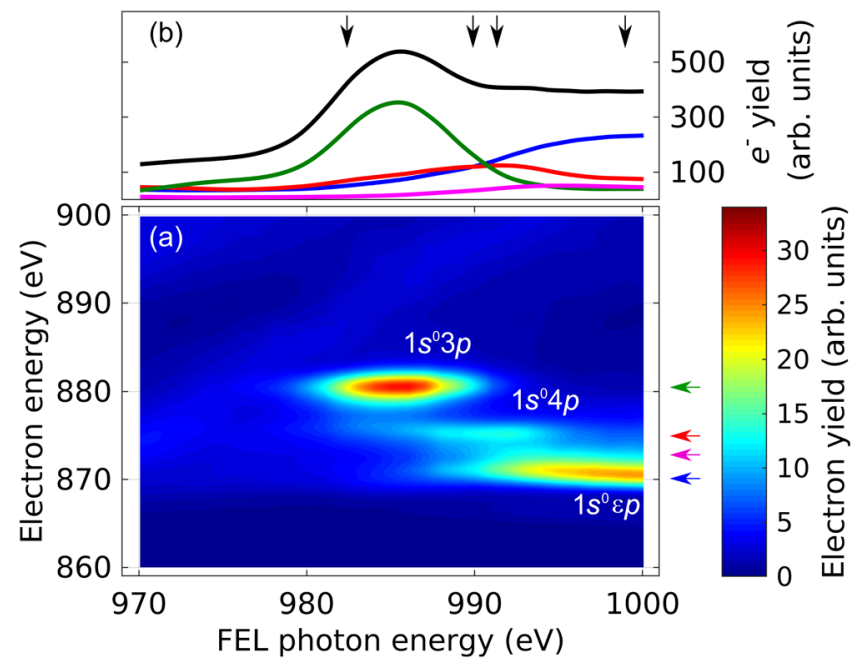

FIG. 2. Resonance map of the transient Ne ion. (a) Interpolated false color representation of the electron spectra recorded by varying the photon energy across the region of the $\mathrm{Ne}^{+} 1 s^{1} 2 s^{2} 2 p^{6} \rightarrow 1 s^{0} 2 s^{2} 2 p^{6} n p$ transitions with steps of $1 \mathrm{eV}$. (b) Total DCH Auger-electron yield (black line). The total yield is decomposed into individual contributions for the different Rydberg states by projecting the 2D map at selected electron energies [see colored arrows on the right side of (a)]: $1 s^{0} 3 p$ excitation (green, electron energy $E_{k}=881 \mathrm{eV}$ ), $1 s^{0} 4 p$ excitation (red, $E_{k}=875.5 \mathrm{eV}$ ), $1 s^{0} 5 p$ excitation (magenta, $E_{k}=$ $872.5 \mathrm{eV}), 1 s^{0} \varepsilon p$ excitation (blue, $E_{k}=870.5 \mathrm{eV}$ ). The integration regions for the selected electron energies are $6,3,1$, and $0.5 \mathrm{eV}$, respectively. The black arrows indicate the photon energies selected for the detailed analysis of the relaxation pathways (Fig. 3). A small contribution of direct valence photoionization of $\mathrm{Ne}^{2+}$ ions in their ground state can be seen as faint diagonal lines at high electron energies.

energy is within the resonance bandwidth and if the photon is delivered within the lifetime of the core-excited ion. The high intensity and the $\mathrm{kHz}$ pulse delivery repetition rate used in the present experiments could effectively counterbalance these disadvantages and thereby enable the detailed characterization of the $\mathrm{DCH}$ resonances.

Figure 2 shows the recorded electron spectra at different $x$-ray energies covering the range from below the first unoccupied $(n=3)$, over all Rydberg states, into the continuum. Despite the photon-energy bandwidth of about $0.9 \%$, the individual features of the $n=3$ and $n=4$ resonances are clearly identified, whereas higher lying states are increasingly overlapping. The signal at approximately $870-\mathrm{eV}$ electron energy continues at the very same energy for shorter wavelengths since it is a discrete transition in the doubly-core-ionized ion, as reported in the literature for atomic and molecular systems [1,22-28]. An electron spectrum with more details of the accessible features above the $1 s^{-2}$ threshold, which was experimentally determined at an excitation energy of $995 \pm 2 \mathrm{eV}$, is shown in Sec. IV.

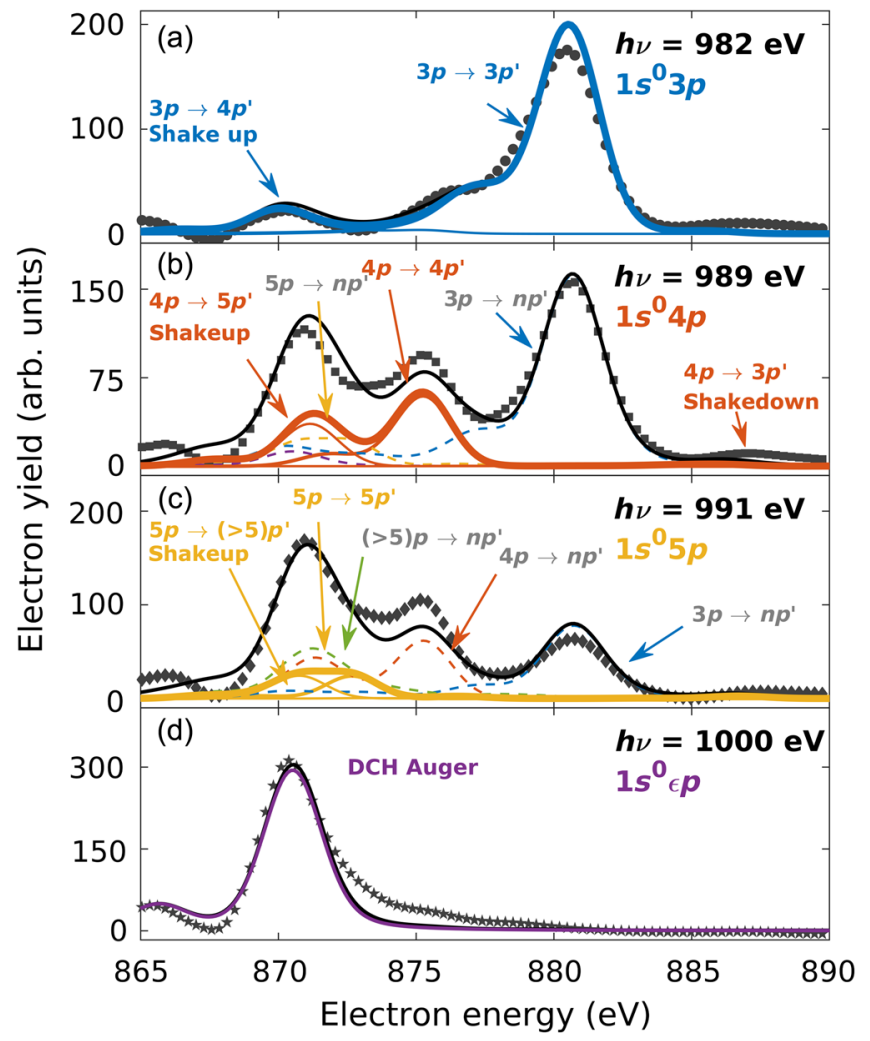

FIG. 3. Electron spectra from individual resonances. The experimental electron spectra at (a) $h \nu=982 \pm 0.5 \mathrm{eV}$ (circles), (b) $h \nu=989 \pm 0.5 \mathrm{eV}$ (squares), (c) $h \nu=991 \pm 0.5 \mathrm{eV}$ (diamonds), and (d) $h \nu=1000 \pm 0.5 \mathrm{eV}$ (stars) are plotted in comparison to the calculated yield from all transitions that are possible within the specified photon-energy bandwidth (thick black curves). For each panel, the color code assigns blue to $3 p$, red to $4 p$, yellow to $5 p$, green to $(>5) p$, and purple to continuum excitation states. The theory spectra are decomposed in the contribution from the photon-energy-selected resonant excitation (full thicker lines) and, for panels (b) and (c), the non-negligible yields from close lying resonances (dashed lines) labeled in gray. The photon-energy-selected excitation yields are further resolved in the contributions attributed to the different final states (full thick lines, spectator decay; full thin lines, shakeup or -down decays).

\section{Discussion}

The interpretation and assignment of the observed structures are enabled by model spectra based on photoionization calculations using the $B$-spline $R$-matrix method [29] in which nonorthogonal electronic orbitals are used to describe the relaxation effects. The calculations are carried out in the $L S$ coupling scheme of the angular momenta, when orbital $(L)$ and spin $(S)$ angular momenta of the electrons are both conserved. All initial and final boundstate orbitals are obtained by multiconfiguration HartreeFock methods (see Sec. IV). Theory-based-model spectra are depicted in Fig. 3 in comparison with the experimental results, showing good overall agreement. The theoretical yields for the resonant Auger electron emission are 
obtained from the calculated transition probabilities accounting for the experimental conditions (see Sec. IV) and are fit to the experimental data by one single scaling parameter for the amplitude of the resonant yields for all photon energies.

For all resonances, the dominant decay channel is related to spectator transitions $\mathrm{Ne}^{+} 1 s^{0} 2 s^{2} 2 p^{6} n p \rightarrow$ $\mathrm{Ne}^{2+} 1 s^{1} 2 s^{2} 2 p^{4} n p^{\prime}\left(n p=n p^{\prime}\right)$. The lowest photon energy [Fig. 3(a)] is chosen to exclusively excite the $1 s^{0} 2 s^{2} 2 p^{6} n p$ $(n=3)$ Rydberg state. The simulated Auger spectrum of the $\mathrm{Ne}^{+} 1 s^{0} 2 s^{2} 2 p^{6} 3 p$ state is decomposed in contributions to the different final states, $\mathrm{Ne}^{2+} 1 s^{1} 2 s^{2} 2 p^{4} 3 p^{\prime}$ (spectator decay labeled as $3 p \rightarrow 3 p^{\prime}$ ) and $\mathrm{Ne}^{2+} 1 s^{1} 2 s^{2} 2 p^{4} 4 p^{\prime}$ (shakeup labeled as $3 p \rightarrow 4 p^{\prime}$ ). The highest photon energy [Fig. 3(d)] lies above the $1 s^{-2}$ threshold and the electron spectrum relates to the normal Auger decay of the doublecore hole. The two middle panels in Fig. 3 show the spectra for photon energies triggering the excitation of the $1 s^{0} 2 s^{2} 2 p^{6} n p(n=4, n=5)$ Rydberg states. Because of the photon-energy bandwidth, these spectra contain, in addition to the selected resonant Auger transition, also a non-negligible contribution from the excitation of other close-lying resonances, which is captured by theory and also represented in Figs. 3(b) and 3(c). The decay of the $1 s^{0} 2 s^{2} 2 p^{6} n p(n=4, n=5)$ resonances is decomposed into the spectator-decay contribution and the shakeup and shakedown contributions labeled in an analogous way to Fig. 3(a).

Identifying final states with quantum numbers different from the excitation level allows for a detailed characterization of the decay pathways from these highly excited resonant states, and to estimate the relevance of the shakeup $\left(n<n^{\prime}\right)$ and shakedown $\left(n>n^{\prime}\right)$ contributions in the relaxation of a specific resonance. For the $3 p$ resonance, we observe a remarkably low shakeup probability, with about $11 \%$ of the total decay found in the $\mathrm{Ne}^{2+} 1 s^{1} 2 s^{2} 2 p^{4} 4 p^{\prime}$ configuration. For the $\mathrm{Ne}^{+} 1 s^{0} 2 s^{2} 2 p^{6} 4 p$ resonance, the contribution from shake processes accounts for $37 \%$ in the $5 p^{\prime}$ shakeup and 3\% in the $3 p^{\prime}$ shakedown states. Compared to single-core-hole resonances [30], the shake processes are contributing significantly less, which can be interpreted by differences in the effective charge for the two processes leading to a different expansion of the $p$ orbitals. At high irradiation levels around $10^{18} \mathrm{~W} / \mathrm{cm}^{-2}$, Rabi cycling may affect the Auger spectrum and population yields for the resonant double-core vacancy $[31,32]$. The shape of the resonant Auger lines starts to be modified when the Rabi oscillation period becomes comparable to the Auger-decay time. The corresponding critical intensity for the $1 s-3 p$ transition in $\mathrm{Ne}^{+}$is estimated to about $10^{19} \mathrm{~W} / \mathrm{cm}^{2}$. This is still above the peak intensity in our experiments, and only small changes in the wings of the resonant Auger lines might occur. The detailed analysis of this effect is beyond the scope of this paper and is neglected in the theoretical modeling.

\section{CONCLUSIONS}

Enabled by the efficient wavelength tunability and the high repetition rate of the European XFEL, we demonstrate the capability to investigate highly transient core-ionized species via electron spectroscopy. The method is promising to evolve as a tool to investigate transient chemical functionality of molecules by studying the electronic structure of, e.g., core-hole-excited systems for understanding electron migration and the evolution of electron correlations on the atto- and femtosecond timescale. It can provide benchmarks for theoretical models of intramolecular charge transfer and nuclear dynamics immediately after the sudden creation of a core hole, which have mainly been assessed indirectly via ion spectroscopy up to now [33]. The presented results allow, furthermore, a much deeper insight into the typically neglected radiation-damage dynamics on the onset of the XFEL pulse, with possible implications for biomolecular imaging applying the "diffract-before-destroy" principle.

\section{METHODS}

\section{A. Experiment}

The experiment is carried out at the SQS scientific instrument located on the SASE3 soft-x-ray branch of the European XFEL [21], using the atomic-like quantum systems (AQS) experimental station [34]. For the present experiment, up to 2000 pulses per second are distributed equally within $200-\mu$ s-long pulse trains at a repetition rate of $10 \mathrm{~Hz}$. The soft x-ray undulators in SASE3 are currently providing linear horizontal polarization, and the photon energy can be quickly tuned via adjusting the gap between the magnetic rows. Using a total electron charge of $250 \mathrm{pC}$, the x-ray pulses have an estimated duration of about $25 \mathrm{fs}$ with pulse energies of about $2 \mathrm{~mJ}$ on average. Their photonenergy bandwidth including a shot-to-shot jitter is experimentally determined to be $0.9 \%$.

Two highly polished focusing mirrors mounted in KB configuration provide a focus size of about $1.5 \mu \mathrm{m}$ diameter (FWHM) and thereby an intensity of up to $3 \times$ $10^{18} \mathrm{~W} / \mathrm{cm}^{2}$ in the interaction volume, taking into account a beam-line transmission of about $56 \%$ at $1000 \mathrm{eV}$ photon energy. Details on the beam-line parameters will be published elsewhere. The optimization of the focal spot with the KB optics is realized using a Hartmann-type wavefront sensor as well as using $\mathrm{Xe}$ and $\mathrm{Ne}$ ion spectrometry. The photon energy is experimentally determined by comparing the resonance energy of the $\mathrm{Ne} 1 s^{-1} 3 p$ excitation, i.e., $867 \mathrm{eV}$, to the corresponding accelerator-based estimation from electron-beam diagnostics. The relative uncertainty for extrapolating the calibration to $1000 \mathrm{eV}$ photon energy is about $1 \mathrm{eV}$.

The employed electron time-of-flight (TOF) spectrometer is installed facing the interaction region of the AQS ultra-high-vacuum chamber where the $\mathrm{x}$-ray focus is 
optimized. It is aligned at an angle of $54.7^{\circ}$ with respect to the horizontal linear polarization of the $\mathrm{x}$ rays, which means that angular distribution anisotropies are negligible. The interaction volume is defined by the x-ray beam and an effusive beam of gaseous neon (99.999\% purity). When introducing the neon gas during the experiment, the pressure in the vacuum chamber rises to about $1 \times 10^{-7}$ mbar, whereas the local pressure in the interaction is estimated to be 1 to 2 orders of magnitude higher. The total acceptance of the TOF is $0.14 \%$ of $4 \pi$. After having passed the entrance of the spectrometer, the electrons enter a $417-\mathrm{mm}$-long flight tube that is divided into four insulated sections, which create a set of static electric fields that successively retard the incoming electrons. Their voltages can be tuned independently to optimize the energy resolution. For the measurements on the resonant DCH Auger lines (Figs. 2 and 3), a retardation voltage of $-850 \mathrm{~V}$ is applied. In counting mode, i.e., with single-electron hits per pulse, the energy resolution is shown to reach up to $E / \Delta E>10000$. The electrons are finally preaccelerated onto a commercially available microchannel-plate (MCP) detector that is operated in analog mode; i.e., the current through the MCPs creates the electron spectrum, which requires multiple electron hits per time bin.

\section{B. Data analysis}

The recorded electron spectra are obtained from the analog current signal of the MCPs collected by the detector anode that is ac coupled to a 12-bit analog-to-digital converter (ADC) with a 2-GHz bandwidth. These measured
$\mathrm{ADC}$ traces are deconvoluted by the response function of the detector in order to obtain a reliable retrieval of the line shape in the high-resolution spectra.

The linearity of the detector response to the collected signal along a 100 to $200 \mu$ s pulse train is characterized using the yield from the single-photon valence photoemission $\mathrm{Ne} 1 s^{2} 2 s^{2} 2 p^{6}+h \nu \rightarrow \mathrm{Ne}^{+} 1 s^{2} 2 s^{1} 2 p^{6}+\varepsilon l$ and $\mathrm{Ne} 1 s^{2} 2 s^{2} 2 p^{6}+h \nu \rightarrow \mathrm{Ne}^{+} 1 s^{2} 2 s^{2} 2 p^{5}+\varepsilon l$ (see Fig. 4), which is applied as a normalization factor.

The signal from the single $\mathrm{x}$-ray pulses is then sorted according to the pulse energy measured by the SASE3 gas monitor detector to extract the dependence of the DCH Auger-electron yield on the pulse energy. The power-law plot (inset in Fig. 4) clearly shows a quadratic dependence on the number of photons of the continuum DCH Auger, which is consistent with the nonlinear nature of the observed process.

The TOF spectrometer is energy calibrated using its time-of-flight to kinetic-energy dependence and its transmission function as extracted for the kinetic-energy region of interest by interpolating the photon-energy dependence of the single-photon valence photoemission yields in neon. The transmission is extracted by normalizing the yield upon the double-differential cross section of the $2 p$ and $2 s$ photoemission processes. In the photon-energy region where the resonant excitation takes place, the resonant Auger signal superposes to the valence photoemission from $\mathrm{Ne}^{2+} 1 s^{2} 2 s^{n} 2 p^{m}(n+m=6)$ ions, whose kinetic energy varies with the photon energy. In order to be able to perform a quantitative analysis of the resonant Auger spectra, this

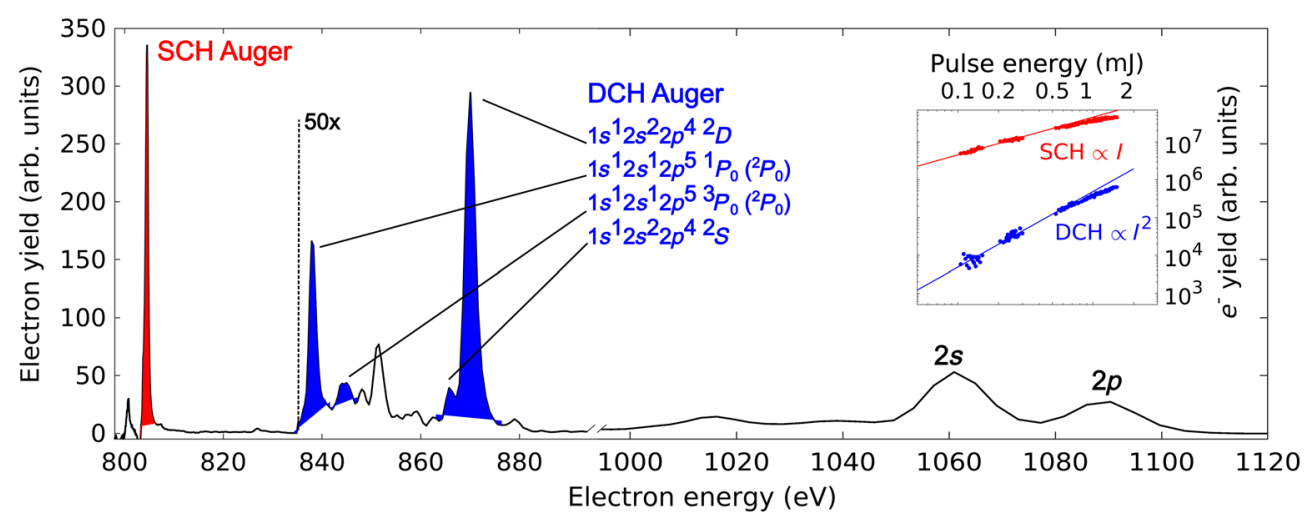

FIG. 4. Electron spectrum above the DCH ionization threshold. High-resolution electron spectrum of neon ionized at $h v=1110 \mathrm{eV}$ with a pulse energy of $1.1 \pm 0.1 \mathrm{~mJ}$. The sharp feature at an electron energy $804.5 \mathrm{eV}$ shaded in red is attributed to the $\mathrm{KL}_{23} L_{23}\left({ }^{1} D\right)$ Auger line generated by the decay of the $\mathrm{SCH}$ state $\mathrm{Ne}^{+} 1 s^{1} 2 s^{2} 2 p^{6}$ after $1 s$ ionization of the neutral. The broad structures at 1060 and $1090 \mathrm{eV}$ result from the valence ionization of the neutral atom. The structure between 835 and 875 eV electron energy is mostly assigned to the DCH Auger decay, with final states as indicated in the legend. The spectroscopic assignments are taken from Ref. [24] and references therein. The two uncolored lines around $850 \mathrm{eV}$ may be identified according to Ref. [24] as decays $\mathrm{Ne}^{2+} 1 s^{0} 2 s^{2} 2 p^{5} 3 s, 3 p \rightarrow \mathrm{Ne}^{3+} 1 s^{1} 2 s^{1} 2 p^{4} 3 s, 3 p$, which are excited from the ground state of Ne by two photons much stronger than by a single photon of the synchrotron radiation. Inset: pulse energy dependence of the SCH (red) and DCH (blue) contributions over more than an order of magnitude obtained by attenuating the pulse energy upstream the interaction region. The dots represent the experimental data obtained by integrating the electron yield from the shaded areas in the spectrum at given pulse energies; the lines represent power-law fits from the pulse energy range $\mathrm{E}_{\text {pulse }}<800 \mu \mathrm{J}$, where the experiment is not affected by target depletion. The extracted power laws are consistent with a one-photon (linear) and a two-photon process, respectively. 
contribution is modeled for the spectrum collected at $h \nu=1000 \mathrm{eV}$, where no superposition with the $\mathrm{DCH}$ Auger yield is observed. It is then subtracted from the photon-energy-dependent spectra after scaling it by the photoionization cross section of neutral $\mathrm{Ne}$.

The theoretical resonant Auger yields to be compared to the experimental spectra are obtained from the calculated transition probabilities as we describe below, accounting for the contribution from the SASE photon-energy bandwidth of $8.5 \mathrm{eV}$ FWHM and convoluting the spectra with the response function of the electron spectrometer $(2.1 \mathrm{eV}$ FWHM in the applied settings).

\section{Photoionization calculations}

The photoionization calculations are performed in the $L S$-coupling approximation with relaxation by the $R$ matrix method with $B$ splines, as realized in the BSR codes [29]. The nonorthogonality between electron wave functions in the initial and final states, which is crucial in treating ionization from the inner shell, is fully taken into account. The electron wave functions for the bound states are obtained in the multiconfiguration Hartree-Fock approximation [35]. For the comparison with the experiment, an overall offset of about $4 \mathrm{eV}$ must be introduced to match the calculated and experimental excitation energies. In addition, the energies of the Rydberg states with different quantum numbers are finally slightly shifted with respect to each other to account for the deviations due to the finite basis set included in the calculations. The photoabsorption cross section of $\mathrm{Ne}^{+} 1 s^{1} 2 s^{2} 2 p^{6}$ for the photon energies covering the region of the $\mathrm{Ne}^{+} 1 s^{0} 2 s^{2} 2 p^{6} n p$ DCH resonances and the $\mathrm{Ne}^{2+} 1 s^{0} 2 s^{2} 2 p^{6}$ ionization threshold is shown in Fig. 5. The following final doubly charged $\mathrm{Ne}^{2+}$ states (thresholds) are included:

$$
\begin{gathered}
1 s^{1} 2 s^{2} 2 p^{5}, 1 s^{1} 2 s^{1} 2 p^{6}, 1 s^{0} 2 s^{2} 2 p^{6}, \\
{\left[1 s^{1} 2 s^{2} 2 p^{4}, 1 s^{1} 2 s^{0} 2 p^{6}, 1 s^{0} 2 s^{2} 2 p^{5},\right.} \\
\left.1 s^{1} 2 s^{1} 2 p^{5}, 1 s^{0} 2 s^{1} 2 p^{6}\right] 3 s / 3 d, \\
{\left[1 s^{1} 2 s^{2} 2 p^{4}, 1 s^{1} 2 s^{1} 2 p^{5}, 1 s^{1} 2 s^{0} 2 p^{6},\right.} \\
\left.1 s^{0} 2 s^{2} 2 p^{5}, 1 s^{0} 2 s^{1} 2 p^{6}\right] n p(3 \leq n \leq 8),
\end{gathered}
$$

giving in total 321 states with different total orbital and spin angular momenta. Strong resonances from the DCH states dominate the spectrum below the threshold and, as we demonstrate in Fig. 5, the width of these resonances is determined by the decays within the ionic core with the spectator Rydberg $n p$ electron. The calculated lifetimes are $0.89 \mathrm{fs}$ (corresponding to $740 \mathrm{meV}$ ) for the $3 p$ resonance and $1.02 \mathrm{fs}(650 \mathrm{meV})$ for the $4 p$ resonance. Our model goes beyond the second-order perturbation theory calculations in Ref. [36] for the similar process in the energy range under consideration.

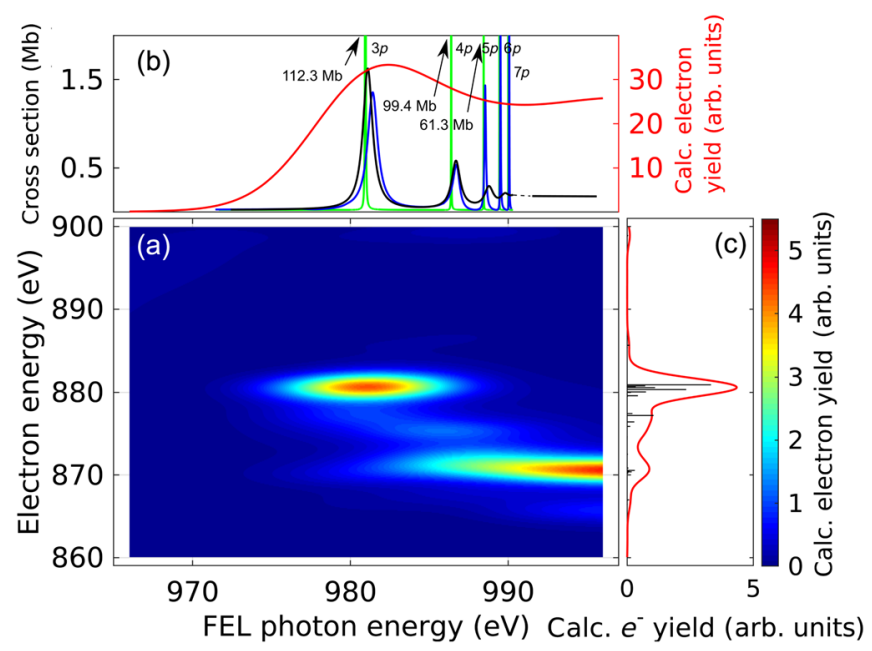

FIG. 5. Calculated photoabsorption cross section, resonant Auger spectrum, and resonance map of the transient $\mathrm{Ne}$ ion. (a) Interpolated false color representation of the theory-based electron spectra for different photon energies across the region of the $\mathrm{Ne}^{+} 1 s^{1} 2 s^{2} 2 p^{6} \rightarrow \mathrm{Ne}^{+} 1 s^{0} 2 s^{2} 2 p^{6} \mathrm{np}$ transitions. The map results from the convolution of the calculated photon-energydependent electron spectra with a two-dimensional Gaussian of widths taken from the experimental electron spectrum resolution $2.1 \mathrm{eV}$ FWHM and the photon-energy bandwidth of $8.5 \mathrm{eV}$ FWHM, as given in the text. (b) Left axis: calculated cross section of the transient $\mathrm{Ne}$ ion. Green: without inclusion of $n p$-spectator decay channels. Blue: with inclusion of decay channels related to $3 p$ and $4 p$ spectator electrons. Black: with inclusion of all decay channels listed in the text. The scale for the right axis is the cross section in arbitrary units for the red solid line, which is obtained after convolution with an $8.5-\mathrm{eV}$-wide Gaussian. This line corresponds to the integral over the kinetic energy axis of the 2D map shown in (a). (c) Black: calculated resonant Auger spectrum at a photon energy of $981 \mathrm{eV}$ corresponding to the calculated excitation energy of the $3 p$ resonance. Red: the same spectrum after convolution with both the photon-energy bandwidth and the spectrometer response function. This line corresponds to a column at a given photon energy in the 2D map shown in (a).

We simulate the emission lines of the normal Auger decay [Fig. 3(d)] by the same $R$-matrix code, which treats single-photon ionization, considering it as the resonant Auger decay of the $\mathrm{Ne}^{2+} 1 s^{0} 2 s^{2} 2 p^{6}$ state,

$$
\begin{aligned}
& h \nu(\text { XFEL })+\mathrm{Ne}^{2+} 1 s^{1} 2 s^{2} 2 p^{5} \\
& \quad \rightarrow \mathrm{Ne}^{2+} 1 s^{0} 2 s^{2} 2 p^{6} \rightarrow \mathrm{Ne}^{3+}+e_{A} .
\end{aligned}
$$

The channels with the final configurations $\mathrm{Ne}^{3+} 1 s^{1} 2 s^{1} 2 p^{5}, 1 s^{1} 2 s^{2} 2 p^{4}, 1 s^{1} 2 s^{0} 2 p^{6}$ are included to describe the width of the Auger line, in addition to the $\mathrm{Ne}^{3+} 1 s^{0} 2 s^{2} 2 p^{6}$ threshold of the Auger series $\mathrm{Ne}^{2+}$ $1 s^{0} 2 s^{2} 2 p^{5} \mathrm{np}(n=2,3, \ldots, \infty)$. The relative intensity of the line in Fig. 3(d) is a fitting parameter. 
The experimental data were collected during users beamtime 2193 at the European XFEL. The metadata are available at [37].

\section{ACKNOWLEDGMENTS}

We acknowledge European XFEL in Schenefeld, Germany for provision of the X-ray free-electron laserbeam time at the SQS instrument and would like to thank the staff for their assistance. M. Me., M. Ma., and T. M. acknowledge support by the SFB 925, Project A3, of the University of Hamburg. M. I., V. M., and Ph. S. acknowledge funding from the Volkswagen Foundation within a Peter Paul Ewald Fellowship. E. V. G. and M. D. $\mathrm{K}$. acknowledge funding from the Foundation for the Advancement of Theoretical Physics and Mathematics BASIS under the program "Junior Leader," A. N. G., E. V. G., and M. D. K. acknowledge funding by RFBR under the Research Project No. 20-51-45001. O. Z. acknowledges support from the United States National Science Foundation under Grant No. PHY-1520970. The research is partly carried out using the equipment of the shared research facilities of HPC computing resources at Lomonosov Moscow State University. N. B. acknowledges funding by the Chemical Sciences, Geosciences and Biosciences Division, Office of Basic Energy Sciences, Office of Science, U.S. Department of Energy Grant No. DE-SC0012376. P. J. acknowledges support from the Swedish Research Council and the Swedish Foundation for Strategic Research. S.P. and D. R. are supported by the Chemical Sciences, Geosciences, and Biosciences Division, Office of Basic Energy Sciences, Office of Science, U.S. Department of Energy, Grant No. DE-FG02-86ER13491.

T. M., M.I., and M. Me. conceived the experiment, which was carried out by T.M., M. I., T.M.B., R.B., A. D. F., P. G., J. M., V. M., Y.O., D. Ri., Ph. S., N. B., B. E., P. J., C. K.-W., L. M., M. Ma., C. O., S.P., T.P., D. Ro., and M. Me. Installation and commissioning of the SQS instrumentation was done by T. M. B., R. B., A. D. F., P. G., M. I., T. M., J. M., V. M., Y. O., N. R., D. Ri., D. Ro., P. S., R. W., P.Z., and M. Me. The data were analyzed by T. M. Theoretical modeling was performed and elaborated by M. K., E. G., O.Z. and A. N. G. The manuscript was written by T.M., M. I., and M. Me., with contributions from all authors.

[1] L. Young et al. Femtosecond Electronic Response of Atoms to Ultra-Intense X-Rays, Nature (London) 466, 56 (2010).

[2] B. Rudek et al. Ultra-Efficient Ionization of Heavy Atoms by Intense X-Ray Free-Electron Laser Pulses, Nat. Photonics 6, 858 (2012).
[3] A. Rudenko et al. Femtosecond Response of Polyatomic Molecules to Ultra-Intense Hard X-Rays, Nature (London) 546, 129 (2017).

[4] H. Stapelfeldt, E. Constant, H. Sakai, and P. B. Corkum, Time-Resolved Coulomb Explosion Imaging: A Method to Measure Structure and Dynamics of Molecular Nuclear Wave Packets, Phys. Rev. A 58, 426 (1998).

[5] E. P. Kanter, B. Krässig, Y. Li, A. M. March, P. Ho, N. Rohringer, R. Santra, S. H. Southworth, L. F. DiMauro, G. Doumy et al. Unveiling and Driving Hidden Resonances with High-Fluence, High-Intensity X-Ray Pulses, Phys. Rev. Lett. 107, 233001 (2011).

[6] B. Erk et al. Imaging Charge Transfer in Iodomethane upon X-Ray Photoabsorption, Science 345, 288 (2014).

[7] P. J. Ho et al. The Role of Transient Resonances for Ultra-Fast Imaging of Single Sucrose Nanoclusters, Nat. Commun. 11, 167 (2020).

[8] S. E. Canton et al. Toward Highlighting the Ultrafast Electron Transfer Dynamics at the Optically Dark Sites of Photocatalysts, J. Phys. Chem. Lett. 4, 1972 (2013).

[9] H. Öström et al. Probing the Transition State Region in Catalytic CO Oxidation on Ru, Science 347, 978 (2015).

[10] T. Brixner, J. Stenger, H. M. Vaswani, M. Cho, R. E. Blankenship, and G. R. Fleming Two-Dimensional Spectroscopy of Electronic Couplings in Photosynthesis, Nature (London) 434, 625 (2005).

[11] H. N. Chapman et al. Femtosecond Diffractive Imaging with a Soft-X-Ray Free-Electron Laser, Nat. Phys. 2, 839 (2006).

[12] K. Ueda et al. Roadmap on Photonic, Electronic and Atomic Collision Physics: I. Light-Matter Interaction, J. Phys. B 52, 171001 (2019).

[13] S. H. Southworth, E. P. Kanter, B. Krässig, L. Young, G. B. Armen, J. C. Levin, D. L. Ederer, and M. H. Chen, Double K-Shell Photoionization of Neon, Phys. Rev. A 67, 062712 (2003).

[14] A. Müller et al. Photoionization of Ne Atoms and $\mathrm{Ne}^{+}$Ions near the K Edge: Precision Spectroscopy and Absolute Cross-Sections, Astrophys. J. 836, 166 (2017).

[15] A. Rouzée et al. Angle-Resolved Photoelectron Spectroscopy of Sequential Three-Photon Triple Ionization of Neon at $90.5 \mathrm{eV}$ Photon Energy, Phys. Rev. A 83, 031401(R) (2011).

[16] M. Braune et al. Electron Angular Distributions of Noble Gases in Sequential Two-Photon Double Ionization, J. Mod. Opt. 63, 324 (2016).

[17] M. Ilchen et al. Symmetry Breakdown of Electron Emission in Extreme Ultraviolet Photoionization of Argon, Nat. Commun. 9, 4659 (2018).

[18] L.S. Cederbaum, F. Tarantelli, and A. Sgamellotti, On Double Vacancies in the Core, J. Chem. Phys. 85, 6513 (1986).

[19] F. Gel'mukhanov and H. Agren, Resonant X-Ray Raman Scattering, Phys. Rep. 312, 87 (1999).

[20] O. Travnikova et al. Subfemtosecond Control of Molecular Fragmentation by Hard X-Ray Photons, Phys. Rev. Lett. 118, 213001 (2017).

[21] W. Decking et al. First Operation of a MHz-Repetition-Rate Hard X-Ray Free-Electron Laser Driven by a Superconducting Linear Accelerator, Nat. Photonics 14, 391 (2020). 
[22] N. Berrah et al. Double-Core-Hole Spectroscopy for Chemical Analysis with an Intense X-Ray Femtosecond Laser, Proc. Natl. Acad. Sci. U.S.A. 108, 16912 (2011).

[23] P. Salén, P. van der Meulen, H. T. Schmidt, R. D. Thomas, M. Larsson, R. Feifel, M. N. Piancastelli, L. Fang, B. Murphy, T. Osipov et al. Experimental Verification of the Chemical Sensitivity of Two-Site Double Core-Hole States Formed by an X-Ray Free-Electron Laser, Phys. Rev. Lett. 108, 153003 (2012).

[24] L. J. Frasinski et al. Dynamics of Hollow Atom Formation in Intense X-Ray Pulses Probed by Partial Covariance Mapping, Phys. Rev. Lett. 111, 073002 (2013).

[25] G. Goldsztejn et al. Double-Core-Hole States in Neon: Lifetime, Post-Collision Interaction, and Spectral Assignment, Phys. Rev. Lett. 117, 133001 (2016).

[26] S. Carniato et al. Single Photon Simultaneous K-Shell Ionization and $K$-Shell Excitation. I. Theoretical Model Applied to the Interpretation of Experimental Results on $\mathrm{H}_{2} \mathrm{O}$, J. Chem. Phys. 142, 014307 (2015).

[27] P. Lablanquie, F. Penent, and Y. Hikosaka, Multi-Electron Coincidence Spectroscopy: Double Photoionization from Molecular Inner-Shell Orbitals, J. Phys. B 49, 182002 (2016).

[28] S. Carniato, Theoretical Simulation of $K-2 V$ Inner-Shell Processes in $\mathrm{Ne}$ and $\mathrm{Ar}$, J. Electron Spectrosc. Relat. Phenom. 239, 146931 (2020).
[29] O. Zatsarinny, BSR: B-spline atomic R-matrix codes, Comput. Phys. Commun. 174, 273 (2006).

[30] A. Kivimäki, S. Heinäsmäki, M. Jurvansuu, S. Alitalo, E. Nõmmiste, H. Aksela, and S. Aksela Auger Decay at the $1 s^{-1} n p(n=3-5)$ Resonances of Ne, J. Electron Spectrosc. Relat. Phenom. 114, 49 (2001).

[31] N. Rohringer and R. Santra, Resonant Auger Effect at High X-Ray Intensity, Phys. Rev. A 77, 053404 (2008).

[32] Y.-P. Sun, Z. Rinkevicius, C. K. Wang, S. Carniato, M. Simon, R. Taieb, and F. Gelmukhanov, Two-PhotonInduced X-Ray Emission in Neon Atoms, Phys. Rev. A 82, 043430 (2010).

[33] R. Boll et al., Charge Transfer in Dissociating Iodomethane and Fluoromethane Molecules Ionized by Intense Femtosecond X-Ray Pulses, Struct. Dynam. 3, 043207 (2016).

[34] T. Tschentscher et al. Photon Beam Transport and Scientific Instruments at the European XFEL, Appl. Sci. 7, 592 (2017).

[35] C. Froese Fischer, T. Brage, and P. Jönsson, Computational Atomic Structure: An MCHF Approach (IOP Publishing, Bristol, 1997).

[36] S. A. Novikov and A. N. Hopersky, Two-Photon KnockingOut of Two Electrons of the $1 s^{2}$-Shell of the Neon Atom, J. Phys. B 35, L339 (2002).

[37] https://doi.org/10.22003/XFEL.EU-DATA-002193-00 